\title{
El juego modificado, recurso metodológico en el fútbol de iniciación Modified game, methodological resource in soccer initiation
}

\author{
Jaime Serra Olivares, Luis Miguel García López, David Sánchez-Mora Moreno \\ Universidad Castilla-La Mancha
}

Resumen: En las últimas tres décadas la enseñanza de los deportes en general y del fútbol en particular, ha progresado de una metodología basada en el modelo tradicional orientado a la técnica, a una perspectiva más flexible y adaptable a las necesidades del jugador, caracterizada por la utilización de juegos modificados. El problema es, que a pesar de la cantidad de propuestas existentes, la investigación no ha justificado empíricamente la utilización de unas u otras en función del contexto de enseñanza-aprendizaje concreto de los aprendices. Este aspecto nos hace preguntarnos qué sentido tiene utilizar el juego modificado como recurso metodológico en la enseñanza de los deportes. En este sentido, hemos observado que el juego modificado se presenta como una herramienta idónea y eficiente para el desarrollo y evaluación del conocimiento y el rendimiento de juego de jóvenes jugadores, y también para el desarrollo de otras dimensiones del aprendizaje. Sin embargo, no se establecen unas directrices justificadas a seguir en su planificación y utilización, motivo por el cual subrayamos la importancia de continuar la investigación en esta línea.

Palabra clave: juego modificado, enseñanza de los deportes, fútbol, rendimiento de juego.

Abstract: In the past three decades teaching sports has changed from a traditional technique-oriented model to teaching games for understanding model characterized by the use of modified games. Despite the number of proposals, research has not empirically justified the use of them depending on the specific teaching-learning context of the practitioners. This way, what function has the modified game as a methodological resource in sports teaching? In this regard, we noted that modified games could be a suitable and efficient tool for development and evaluation of knowledge and game performance of young players, and also for the development of other dimensions of learning. However, there are no guidelines in modified games planning, which is why we stress the importance of continued research in this line.

Key words: modified game, teaching sports, soccer, game performance.

\section{Introducción}

El rendimiento de juego de un deportista, es decir, cómo actúa durante su participación en el entrenamiento o la competición, se debe entre otros factores, al conocimiento previo que posee y a su capacidad para procesar nueva información. A su vez, estos factores se ven influenciados por otros, entre los cuales destacan el papel del entrenador, la experiencia y el contexto de aprendizaje (Greháigne, Godbout, y Bouthier, 2001; Raab, 2007).

En un deporte de invasión como el fútbol, en el que las situacione de juego cambian constantemente, se sugiere que el jugador posee un conocimiento previo (declarativo) sobre las reglas, las posiciones de los jugadores en el campo, que trasciende en su aptitud para relacionar y aplicar nuevos conceptos, es decir, en el qué, el cómo y el por qué de aplicar una conducta, lo que se conoce como conocimiento procedimental (Griffin, Dodds, Placek y Tremino, 2001; Placek y Griffin, 2001).

Ésta afirmación se fundamenta en las teorías constructivistas y de procesamiento de la información, las cuales permiten analizar cómo refina su aprendizaje el jugador estructurando el conocimiento para actuar estratégicamente durante el juego (Contreras, De la Torre y Velázquez, 2001). En este sentido, si tenemos en cuenta que la cantidad de conocimiento declarativo y procedimental, al igual que el factor motor son determinantes sobre el rendimiento de juego del jugador (French y Thomas, 1987; Memmert y Roth, 2007; Nevett, Rovegno y Baviarz, 2001), sería interesante saber de los recursos didácticos de los que dispone un profesor/entrenador para hacer de su práctica un contexto de enseñanza idóneo orientado a la mejora de esos aspectos.

No obstante, la enseñanza de los deportes se ha limitado a la enseñanza de un conjunto de habilidades o tareas aisladas, claramente orientadas a la adquisición de unas técnicas vinculadas al rendimiento que exigen los patrones dominantes del deporte competitivo. Esto se debe principalmente a dos razones: la resistencia al cambio de los profesionales de la Educación Física y la enorme influencia que han ejercido las instituciones deportivas nacionales (Devís y Peiró, 1992).

Fecha recepción: 27-05-11 - Fecha envío revisores: 06-06-11 - Fecha de aceptación: 09-06-11 Correspondencia: Jaime Serra Olivares

Plaza de la Universidad 3

02071, Albacete

E-mail: Jaime.serra@uclm.es
En este sentido, desde la aparición del concepto predeporte como aspecto clave en la enseñanza de los deportes se han elaborado multitud de aportaciones, que sin embargo, no han tenido en cuenta el trabajo realizado por sus predecesores. Éste hecho, ha desembocado en la construcción de propuestas metodológicas que rompen con la orientación mecanicista y técnica dominante del entrenamiento, que teniendo similitud en su ideología, están lejos de un constructo común más enriquecedor (Alarcón, Cárdenas, Miranda, Ureña, Piñar, 2010; Devís y Peiró, 1992; González, García, Contreras y Sánchez-Mora, 2009).

Fi En cualquier caso, podemos apreciar que en las últimas tres décadas la enseñanza de los deportes ha progresado de una metodología basada en el modelo de enseñanza tradicional, orientado a la técnica, a una perspectiva más flexible y adaptable a las necesidades del jugador, en la que se le brinda la posibilidad de construir su propio aprendizaje (Thorpe, Bunker y Almond, 1986).

Si observamos a fondo la estructura actual de las propuestas de enseñanza alternativa de los deportes, se contempla el concepto de iniciación deportiva desde un modelo de enseñanza comprensivo caracterizado por el aprendizaje contextual. Así, se plantea al aprendiz un continuo de juegos modificados o situaciones reducidas del juego real de competición, en las que la incertidumbre requiere que se perciba y analice la situación, se decida cuál es el comportamiento técnico-táctico más adecuado a la misma, y por último, se desarrolle.

Pongamos como ejemplo una situación concreta en el deporte del fútbol, en la que un jugador atacante con balón avanza hacia la portería contraria. En ésta situación, el jugador percibe y analiza el contexto observando la distancia a la que se encuentra la portería y los compañeros, el número de adversarios que defienden, el tipo de defensa o marcaje que realizan. Posteriormente, el jugador procesa la información, delibera su comportamiento técnico-táctico y decide desarrollar una habilidad de juego, por ejemplo, fijar a un defensa atrayendo su atención mientras conduce el balón, realizar un pase a un compañero que se encuentra en una posición más adelantada respecto a la portería contraria, o simplemente realizar un golpeo hacia la misma, y, por último, decidir con qué pierna desarrollar el gesto técnico, la fuerza de contacto con el balón.

En esta línea, si nos encontramos formando jóvenes deportistas y tenemos un objetivo técnico-táctico concreto como puede ser la creación de espacios en ataque, deberíamos saber cuál es la mejor opción para abordar su aprendizaje. ¿Sería más adecuado proponer un juego analítico 
de pase y desplazamiento sin oposición, o plantear un juego de conservación del móvil en el que el punto se consigue cuando un jugador atacante recibe el mismo en una zona concreta?

$\mathrm{Si}$ analizamos las propuestas teóricas relacionadas con el modelo de enseñanza comprensiva de los deportes, estas no sólo se centran en la enseñanza de cómo hacer un gesto técnico, sino que desarrollan una metodología de enseñanza contextual centrada también en el aprendizaje del qué, el por qué y el cuándo de un comportamiento técnico-táctico (Bayer, 1992; Bunker y Thorpe, 1982; Griffin, Mitchell y Oslin, 1997; Lasierra, 1990; Lasierra y Lavega, 1993; Launder y Piltz, 2006; Light, 2006).

El problema es, que a pesar de la cantidad de propuestas existentes, la investigación no ha justificado empíricamente la utilización de unas u otras en función del contexto de enseñanza-aprendizaje concreto de los aprendices (Raab, 2007). Es más, esta situación nos hace preguntarnos qué sentido tiene utilizar el juego modificado como recurso metodológico en la enseñanza de los deportes. $\mathrm{Y}$ es que todavía no se conoce con certeza qué tipo de juego modificado es más adecuado y eficiente, y cuál es su alcance sobre otros objetivos del proceso de enseñanza-aprendizaje de los deportes, como pueden ser las dimensiones afectiva y fisiológica. En el caso del fútbol de iniciación, por ejemplo, observamos un número limitado de estudios que analizan directa o indirectamente el efecto de la utilización del juego modificado como recurso metodológico (Blomqvist, Väntinen y Lhutanen, 2005; Holt, Ward y Wallhead, 2006; Lapresa, Amatria, Egüén, Arana y Garzón, 2008; Memmert y Roth, 2007; Memmert, 2010; Nevett et al., 2001), y se tiende a investigar al jugador adulto, experto, y en otros deportes.

Este entramado finaliza en el establecimiento de unas etapas de aprendizaje, entrenamientos y competiciones aparentemente formativas, que se basan en la adecuación de la complejidad técnico-táctica del juego a la edad de los participantes, y no tanto a su nivel de conocimientos y habilidades (Fradua Uriondo, 1999; Lago, 2002; Mitchell, Griffin y Oslin, 2003; Sans y Frattarola, 1996, 1998, 2009; Wein, 1995).

Además, las formas jugadas utilizadas en estas propuestas se limitan a juegos en los que se modifican elementos estructurales y organizativos como pueden ser el tiempo, el espacio o el número de jugadores que participan. Sin embargo, ¿qué es lo que define la problemática contextual del juego? ¿Qué elementos del deporte condicionan las decisiones a tomar por los jugadores en función del contexto de juego concreto en el que se encuentran? Estos son, los principios de actuación de los juegos/ deportes de invasión (Bayer, 1992).

No tener presente estos principios, así como otras posibilidades de modificación deportiva (Bunker y Thorpe, 1982), puede dar lugar a que los aprendices tengan que practicar situaciones para las cuales no han sido formados y en las que probablemente se esté limitando su aprendizaje. Esto se observa al analizar algunas modalidades reducidas de iniciación al fútbol, en las que se percibe la dificultad de los jugadores para adaptarse en amplitud y profundidad a los problemas de juego (Lapresa et al., 2008; Lapresa, Arana, Garzón, Egién y Amatria, 2010).

De ahí la importancia de conocer la función y el significado de los principios de actuación en los juegos y deportes de invasión. De ahí el valor del juego modificado en el proceso de enseñanza-aprendizaje de los juegos y deportes. Por este motivo, y como primera aproximación al tema, que el objetivo de este trabajo sea revisar el estado de la literatura en torno a la utilización del juego modificado como recurso metodológico en el fútbol de iniciación.

\section{El fútbol, un deporte de invasión}

Si analizamos la naturaleza de los juegos y deportes de invasión, entre los que se encuentra el fútbol, notaremos que existe una similitud patente en las decisiones a tomar durante el juego, y es que todas ellas se realizan en función a unos principios tácticos de actuación (Tabla 1). Podríamos definir el concepto de principio de actuación como una serie de fórmulas que el jugador debe tener en cuenta en función del problema de juego al que se está enfrentando. Estos principios son el conjunto de la interacción de las capacidades de percepción, toma de decisiones y

\begin{tabular}{|c|c|}
\hline Tabla 1. Principios tácticos de actuación de los juegos/deportes de invasión (Bayer, 1992) \\
\hline Ataque & Defensa \\
\hline Conservación del balón & Recuperación del balón \\
\hline $\begin{array}{c}\text { Avance de los jugadores y del balón hacia } \\
\text { la meta contraria }\end{array}$ & $\begin{array}{c}\text { Impedir la progresión de los jugadores y del } \\
\text { balón hacia mi portería }\end{array}$ \\
\hline $\begin{array}{c}\text { Ataque a la meta contraria, es decir, marcar } \\
\text { un punto }\end{array}$ & Protección de mi meta o campo \\
\hline
\end{tabular}

ejecución técnico-táctica. Bayer (1992:52) afirma que «estos principios constituyen el punto de partida, la base; representan la fuente de la acción, definen las propiedades invariables sobre las cuales se realizará la estructura fundamental del desarrollo de los acontecimientos».

En los últimos años, estudios como los de Blomqvist et al. (2005); González Villora, García López, Contreras y Gutiérrez (2010), González Víllora, García López, Pastor y Contreras (2010) han analizado el rendimiento de juego del joven futbolista y han llegado a la conclusión de que es recomendable desarrollar un tratamiento contextualizado de los principios tácticos básicos en las fases de iniciación a los deportes, como vienen proponiendo algunos autores en sus progresiones didácticas (Contreras et al., 2001; Griffin et al., 1997). Sin embargo, no está muy claro qué tratamiento de los principios de actuación es el más adecuado para el correcto aprendizaje de un deporte en cuestión.

En el deporte del fútbol por ejemplo, algunos autores como Mitchell et al. (2003) propusieron una enseñanza comprensiva mediante juegos modificados en torno a unos principios tácticos que no coincidían exactamente con los planteados por Bayer (1992). Un ejemplo es el hecho de que los movimientos con balón planteados por estos autores se encuentran dentro de la penetración y el ataque, relacionado principalmente con el tercer principio planteado por Bayer, conseguir elgol.

Sin embargo, si analizamos una jugada como la ejemplificada al inicio de este trabajo, observamos que el jugador también puede conservar la posesión del balón o avanzar hacia la portería contraria realizando movimientos con balón. En cualquier caso, parece ser que Mitchell y colaboradores no han realizado ningún estudio que sustente esta progresión en la enseñanza del fútbol.

De igual modo, si revisamos las propuestas de otros autores como Fradua Uriondo (1999), el tratamiento de los principios tácticos es muy diferente. Este autor, por ejemplo, propone como objetivos tácticos en ataque para jugadores de 8-10 años entender los principios básicos (conseguir gol e impedirlo), y para jugadores de 10-12 años comprender la función del oponente actuando en consecuencia. Es decir, que en primer lugar se podrían utilizar juegos modificados para aprender el tercer principio de actuación en ataque propuesto por Bayer, conseguir gol y después practicar juegos para los otros dos principios indistintamente, conservar la posesión del balón o avanzar hacia la portería contraria.

Por otro lado, Contreras et al. (2001) utiliza como criterio de progresión en su propuesta los principios tácticos planteados por Bayer y el tipo de comunicación que se realiza en el juego modificado (cooperación, oposición o cooperación-oposición). De manera que para estos autores, sería recomendable utilizar juegos modificados sin oposición y gradualmente aumentar la complejidad del principio táctico aumentando el número de jugadores. Por tanto se podría comenzar realizando juegos individuales y juegos de conservación del balón de 1 contra 1,2 contra 1,2 contra $2 \ldots$ para después realizar juegos modificados de avanzar hacia la meta contraria y por último marcar gol.

En esta línea, Wilson (2002) planteó un tratamiento similar para los juegos y deportes de invasión. Su propuesta, se basaba en una reorganización continua del conocimiento de los jugadores. Así, un jugador podría comenzar a aprender un módulo de contenidos técnicotácticos de forma independiente, teniendo en cuenta comobase el módulo anterior. En este sentido, es importante mencionar que los objetivos planteados por este autor coinciden con los de Bayer, pero con un tratamiento diferente de los principios tácticos de actuación, de forma similar a Griffin et al. $(1997,2003)$. Sin embargo, Wilson no indicó la edad de comienzo de cada uno de los módulos de aprendizaje, ni tampoco 
la duración de las etapas. Estos aspectos nos hacen preguntarnos qué principio táctico sería más adecuado desarrollar primero y de qué modo sería más significativo hacerlo.

En cuanto a la propuesta de Griffin et al. (1997), nos parece importante resaltar la recomendación de no introducir la enseñanza de la técnica hasta que el jugador no perciba la necesidad de aprenderla para desarrollarla en contextos que lo requieran. En este sentido, otras propuestas de enseñanza comprensiva de los deportes como la de Launder y Piltz (2006) plantean una intervención didáctica mediante el entrenamiento de la técnica y la táctica por separado, y restan importancia al conocimiento de las reglas de juego.

Por el contrario, y a pesar de que Light (2006) menciona en su propuesta de iniciación a los deportes la posibilidad de adoptar un enfoque directivo en situaciones concretas de enseñanza de la técnica, coincide con otros autores en el valor educativo y formativo que tiene la práctica de juegos modificados del juegoreal, en los queel descubrimiento guiado es crucial (Contreras et al., 2001; Raab, 2007).

En relación a lo anterior, debemos destacar las propuestas de Wein (1995), Sans y Frattarola $(1996,1998,2009)$ como planteamientos de iniciación al fútbol que utilizan juegos modificados y situaciones contextualizadas del juego real. Wein por ejemplo, plantea una progresión a partir de seis años con los siguientes niveles de formación: a) Juegos de habilidades y capacidades básicas, b) Juegos para el Mini-Fútbol, c) Juegos para el Fútbol A-7, d) Juegos para el Fútbol A-8 y A-9 y e) Fútbol reglamentario. Sin embargo, el criterio para el aumento de la dificultad de sus módulos es de nuevo la modificación deportiva atendiendo principalmente a la complejidad técnica, elnúmero de jugadores o porterías, la mayor velocidad. Y volvemos a notar la carencia de estudios de investigación que sustenten la utilización de este tipo de juegos modificados.

En cuanto a la propuesta de Sans y Frattarola (1996, 1998, 2009), diferencian una serie de niveles a superar por los aprendices o como ellos llaman, programas de aplicación técnica. Estos, parten siempre de situaciones globales de fútbol pero reducidas en tamaños, número de jugadores, modificando reglas etc. Y, si un jugador tuviese problemas en su aprendizaje se utilizarían juegos correctivos de menor dificultad. Sin embargo, estos planteamientos carecen de un criterio sobre el que establecer la progresión del aprendizaje táctico, aspecto que limita la funcionalidad de cara a su aplicación. Esto se observa por ejemplo en la propuesta trimestral de entrenamiento mediante sesiones, en la que identificamos hasta cinco de los seis principios tácticos de actuación propuestos por Bayer (1992)

En relación a estos aspectos, nos parecen muy interesantes dos elementos introducidos por Lago (2002) para la iniciación al fútbol. El primero, es la división de los juegos deportivos colectivos en especialidades segmentarias y funcionales, atendiendo a la inmediatez y/o dilación espacio-temporal en la aparición de problemas de juego. Y el segundo es, el concepto de centro de juego, referente al espacio o zona donde se encuentra el balón.

Así pues, el autor diferencia especialidades segmentarias como el Fútbol A-5 o el baloncesto, en donde los participantes interactúan activamente en el mismo sector del espacio de juego, denominado centro de juego. Y especialidades funcionales como el Fútbol A-11 o el hockey hierba en las que se identifica un espacio de juego de primer nivel (centro de juego de construcción), donde se encuentra el balón y en el que el objetivo para los atacantes es mantener la posesión y avanzar hacia la portería contraria, y para los defensores hacerse con la posesión o evitar el avance del balón. Y un espacio de segundo nivel fuera del centro de juego, en el que no se actúa directamente sobre el balón (centro de juego de equilibrio). En este sentido, se recomienda realizar una iniciación deportiva que permita a los practicantes actuar en el centro de juego o tener posibilidades de incorporarse a él de forma inmediata.

En relación a lo anterior, Lago (2002) propone cinco fases en la iniciación al fútbol en las que aumenta la complejidad atendiendo al tiempo y al espacio. Así, podrían utilizarse en primer lugar juegos modificados denominados episodios de duelo individuales (juegos de 1 contra 1), para después realizar juegos que comprendan las interacciones entre dos o más entornos en los que actúan los jugadores, dentro de la estructura funcional del juego (situaciones de superioridad numérica de 3 contra 1, 4 contra $2 \ldots$ ). Después, relacionar yo-compañero/osadversario-os en situaciones de igualdad numérica. Y por último, una fase o macrosistema de juego en el que se desarrollan las nociones espacio-tiempo y la relación yo-balón-equipo-adversarios.

Al respecto, los principios tácticos a desarrollar serían la penetración y la contención en la segunda fase, la penetración-cobertura ofensiva y contención en la tercera, añadiendo la movilidad, el espacio, la cobertura defensiva, el equilibrio y la concentración en la cuarta y quinta fases. En este sentido, la principal diferencia que podríamos resaltar entre las dos últimas fases es que en la cuarta los objetivos estarían encaminados a elementos técnicos concretos y el entrenamiento del ritmo de juego (velocidad) y en la quinta fase a la circulación del balón y ocupación racional del espacio.

En cualquier caso, lo más relevante de este tipo de iniciación al fútbol es que se utiliza el juego modificado en torno a dos elementos clave para una enseñanza de calidad: el tiempo de compromiso motor y el proceso cognitivo. En este sentido, se acentúa que cualquier actividad no es generadora de aprendizajes, motivo por el cual recae la responsabilidad en el juego modificado como elemento crucial para el aprendizaje de conceptos y competencias asociadas a cada fase evolutiva o momento del aprendizaje. Ahora bien, ¿cuál es el estado de la investigación al respecto?

\section{Posibilidades del juego modificado}

3.1 El juego modificado para practicar problemas de juego similares al juego real

Blomqvist et al. (2005), al analizar el rendimiento de juego de jugadores de fútbol de 14-15 años en tres juegos de fútbol modificados, uno de conservar la posesión del balón, otro de avanzar hacia la meta contraria y otro de conseguir gol, hallaron un número significativamente mayor de decisiones y acciones en el juego de conservar la posesión del balón. Además, al analizar el proceso de toma de decisiones, estos autores afirmaron que las decisiones a tomar con balón eran más fáciles que las decisiones a tomar sin balón. Y también sugirieron que en este contexto, es probable que los entrenadores enfaticen más el componente de la ejecución técnico-táctica que el decisional. Sin embargo, estos autores no diferenciaron el rendimiento de juego en función del principio táctico aplicado, marcado por el contextode juego en el que se encontraba el jugador observado en cada momento.

En cualquier caso, estos resultados son muy relevantes para la enseñanza de los deportes de invasión, y más teniendo en cuenta que las decisiones a tomar durante el juego son tanto o más importantes que las ejecuciones. Imaginemos por ejemplo una jugada en la que dos jugadores atacantes se encuentran frente al portero en posición de conseguir un gol. Si el jugador atacante que no tiene el balón no se situase en una situación óptima para recibir el pase de su compañero, se reducirín enormemente las posibilidades de marcar gol. Por tanto, no solamente nos parece interesante el número de decisiones o ejecuciones que desarrollan los jugadores en un juego determinado, como analizaron los citados autores, sino también el hecho de que se éstas sean adecuadas y exitosas (en relación a los principios tácticos de actuación que marca el contexto de juego).

En este sentido, González Víllora (2008), González Vílora, García López, Contreras et al. (2010) y González Vîlora, García López, Pastor et al. (2010) al observar el rendimiento de juego de jóvenes jugadores en juegos modificados de fútbol ( 3 contra 3, 4 contra 4, 5 contra 5 y 7 contra 7), hallaron mayores porcentajes de ejecuciones y decisiones que se desarrollaban en situaciones de avanzar hacia la meta contraria. Concretamente, González Víllora, García López, Contreras et al. (2010), al analizar el rendimiento de juego de jugadores de 12 años en un juego modificado de fútbol 5 contra 5 , observaron un porcentaje mayor de decisiones con éxito en contextos en los que se debía avanzar hacia la meta contraria, a diferencia de cuando se tenía que conservar la posesión del balón. Por contra, González Víllora, García López, Pastor et al. 
(2010), al analizar el rendimiento de juego de jugadores de 10 años durante su participación en un juego modificado de fútbol 3 contra 3 , hallaron mejores porcentajes de éxito en las decisiones en contextos de conservar la posesión del balón.

Aunque el número de jugadores en los juegos modificados analizados en estos estudios es diferente, además de la edad de los mismos. Los datos obtenidos señalan la importancia que tiene desde una perspectiva didáctica, la utilización del juego modificado por principios tácticos como recurso metodológico para la práctica y aprendizaje de comportamientos técnico-tácticos en el deporte. En este sentido, nos parece fundamental en la iniciación a la fase de ataque, el tratamiento por igual de los roles jugador atacante con balón como jugador atacante sin balón, además de la utilización de hojas de observación durante los entrenamientos para determinar si se están cumpliendo los objetivos buscados con el juego o tarea de aprendizaje utilizado.

$\mathrm{Al}$ respecto, al analizar los datos de estas investigaciones, un entrenador podría plantearse aumentar el número de situaciones de avanzar hacia la meta contraria en sus entrenamientos, puesto que en juegos similares al juego real el mayor porcentaje de jugadas se producen en este principio táctico. Sin embargo, la práctica de juegos modificados similares a la competición no siempre es la mejor estrategia para abordar el aprendizaje. Esto lo podemos observar en cualquiera de las categorías de iniciación a los deportes de invasión, en los que se produce el famoso efecto «racimo o paquete» (Bayer, 1992) y todos los jugadores quieren avanzar con el balón hacia la portería contraria.

En relación a lo anterior, nos parece mucho más interesante utilizar juegos modificados que exageren cualquier principio táctico, en los que se aumente el espacio de juego, y se reduzca el número de participantes y en los que los jugadores tengan que crear y ocupar espacios libres para poder desarrollar jugadas más elaboradas. Al respecto podría utilizarse un juego modificado para avanzar hacia la meta contraria (marcada por una línea imaginaria) y en el que los jugadores no puedan avanzar conduciendo o botando el balón.

3.2 El juego modificado para transferir el conocimiento táctico

En los últimos años continúa el debate sobre cuál es la mejor forma de abordar la enseñanza de los juegos deportivos. Principalmente, existen discrepancias entre la enseñanza comprensiva del deporte que incide en la táctica (el qué hacer), y otra más tradicional centrada en la técnica (el cómo hacer). Ambas perspectivas plantean el qué y el cómo hacer con la principal diferencia que la primera se centra más en el proceso de toma de decisiones de juego.

En este sentido, con la enseñanza comprensiva del deporte se introduce «la sustitución de habilidades», que propone por ejemplo, la enseñanza de habilidades de juego en el fútbol realizando pases con las manos, con el objetivo de potenciar el aprendizaje táctico por transferencia.

El problema reside en que existen pocas evidencias de que ésta perspectiva sea la más apropiada para la enseñanza de los deportes. Algunos autores sugieren que el desarrollo de la técnica se consigue con el trabajo de la técnica y el desarrollo de las habilidades tácticas con el entrenamiento táctico. Sin embargo, hemos observado otros estudios que sugieren la transferencia tanto de habilidades técnicas como de toma de decisiones gracias a la aproximación táctica (García López, Contreras, Penney y Chandler, 2009).

$\mathrm{Al}$ respecto, trabajos relacionados con juegos modificados como el de Holt, Ward y Wallhead (2006) estudiaron la trasferencia de juego de la práctica de situaciones de superioridad numérica en fútbol (3 contra 2 y 2 contra 1) sobre juegos modificados de 4 contra 4 . En concreto, la fase de intervención de este estudio consistía en la práctica de situaciones de 1 contra 1 , juegos de posesión de 4 contra 2, tras lo cual se analizaban las conductas de los jugadores en situaciones de superioridad numérica en contextos de avanzar hacia la meta contraria ( 3 contra 2 y 2 contra 1 más el portero). En este sentido, los resultados sugirieron que el 3 contra 2 podía ser un juego modificado más adecuado para preparar a los jugadores para la práctica del 4 contra 4.

Si bien es cierto que mediante metodologías basadas en situaciones problema se cumplen los requisitos necesarios para la adquisición de un conocimiento fructífero y sofisticado (Thorpe et al., 1986). También lo es el hecho de que las propuestas de enseñanza poseen una multitud de juegos modificados diferentes y variados. Desde esta perspectiva no debemos olvidar que los jugadores, gracias a la instrucción formal y a la práctica, refinan su aprendizaje y estructuran el conocimiento para actuar estratégicamente durante el juego. Por tanto, subrayamos como función de un entrenador al respecto la de seleccionar, implementar y evaluar el tipo de juegos modificados utilizados, ya sean con objetivos de rendimiento a corto plazo o de aprendizaje de habilidades a largo plazo. De esta forma, se podría valorar qué tipo de juegos modificados son más adecuados a cada contexto deportivo, determinado por la edad de los jugadores y su nivel de conocimientos y habilidades.

$3.3 \mathrm{El}$ juego modificado como instrumento de evaluación del rendimiento de juego

Memmert (2010) y Vera, Pino, Romero y Moreno (2007) realizaron estudios en los que se analizó el comportamiento técnico-táctico de jóvenes jugadores en juegos modificados de fútbol, juegos que podrían encajar perfectamente con juegos modificados para exagerar el primer principio de actuación en ataque correspondiente a mantener la posesión del balón en el primero de los estudios citados, y un juego modificado de 2 contra 2 para avanzar hacia la meta contraria en el caso del segundo. En este sentido, los autores en recomiendan la utilización del juego modificado como instrumento de evaluación de la variable estudiada.

En esta línea, Thorpe et al. (1986) establecieron dos tipos de juegos modificados, por representación y por exageración, así como las posibilidades de combinación de los mismos. En el caso del fútbol, los juegos modificados por representación consisten en una versión reducida del juego adulto, como por ejemplo las modalidades de Fútbol A-5 o Fútbol A-7, en los que se reduce el espacio de juego, el tiempo, y en donde siempre se mantiene el carácter competitivo y la esencia del juego real. En cuanto a los juegos modificados por exageración, la modificación sería más notoria. Consiste en exacerbar un aspecto concreto para facilitar su aprendizaje, reduciendo o simplificando cualquier elemento (material, principios de juego, reglas) para el desarrollo del entrenamiento integral de un conjunto de habilidades. Un ejemplo de juego modificado por exageración en fútbol sería el juego de los diez pases, en el que dos equipos se enfrentan y consiguen un punto cuando dan diez pases seguidos sin que el equipo contrario se haga con la posesión del balón.

No obstante, ¿qué ocurre cuando un juego como el de los diez pases no cumple su función? Es decir, cuando un grupo de niños de 8 años juega un 4 contra 4 y no consigue dar más de cuatro pases seguidos ise está cumpliendo el objetivo de aprendizaje? Por un lado, existe la posibilidad de que los niños no sean capaces de dar diez pases seguidos y sin embargo, en un juego por parejas sean perfectos pasadores.

En este sentido, ¿cuál es el objetivo de este juego modificado, enseñar/evaluar el principio de conservar la posesión del balón mediante pases y desmarques? Al respecto ¿qué sentido tiene enseñar a mantener la posesión del balón en un juego sin porterías si el objetivo final es enseñar a jugar en situaciones de juego real en el que si las hay? Además ¿realmente es más fácil jugar un 4 contra 4 para mantener la posesión del balón o por el contrario un 4 contra 4 con porterías?

En un 4 contra 4 para mantener la posesión del balón, los defensores solamente tendrían que prestar atención a dos estímulos, el balón y el contrario, mientras que en un 4 contra 4 con porterías existe un estímulo más, las porterías, lo que podría suponer que los jugadores atacantes tuvieran más posibilidades de realizar pases y desmarques sin oposición directa. En este sentido, la solución a todos estos interrogantes solamente se podría conocer si se realizan estudios al respecto.

Los juegos modificados organizados en las propuestas de enseñanza influyen activamente en el aprendizaje de los deportes, abordando diferentes dimensiones delmismo (Rovegno et al. 2001). En este sentido, conocer al detalle los principios que configuran los juegos deportivos y cómo afectan al desarrollo de las conductas de juego, nos permitiría abordar correctamente el proceso de aprendizaje deportivo. Por tanto, nosotros también recomendamos la utilización del juego modificado como instrumento de evaluación del rendimiento de juego, toma de 
decisiones y ejecución técnico-táctica, siempre y cuando los criterios de evaluación preestablecidos hayan sido fundamentados, lo que supone que el juego modificado resulte en un juicio de valor coherente.

En relación a lo anterior, no tendría sentido por ejemplo, utilizar un rondo en fútbol para valorar la capacidad de los jugadores para identificar y ocupar espacios libres. Por el contrario, sería más adecuado utilizar juegos en los que los jugadores tuvieran que ocupar una zona concreta para poder recibir un pase y así anotar un punto.

\subsection{El juego modificado como demanda física y fisiológica} similar al juego real

Los juegos modificados están integrados en todas las propuestas de enseñanza de los deportes. Por tanto, nuestro deber como investigadores, entrenadores y/o profesores, es analizar cada tarea de aprendizaje utilizada, para poder así contribuir a la construcción de metodologías que convengan a la formación integral de los aprendices, seleccionando para ello unos contenidos adecuados también a cada etapa evolutiva y fase sensible en la que se encuentran.

En este sentido, los juegos modificados y las formas jugadas brindan al jugador un abanico de oportunidades de movimiento que permite, además del desarrollo de las habilidades y competencias propias de su edad, la posibilidad de adherirse deliberadamente a la práctica física gracias a programas deportivos basados en el juego (Gabbett, Jenkins y Abernethy, 2009; Hartwig y Naughton, 2007). Además, los juegos modificados suponen la oportunidad de desarrollar parámetros fisiológicos similares a los requeridos en el juego real, como se observa en el estudio de Farrow, Pyne y Gabbett (2008), en el que se analizó el proceso de toma de decisiones y las demandas físicas del deportista en fútbol australiano.

Por tanto, el juego modificado puede suponer un entrenamiento que requiera demandas similares a las exigidas por el juego real o la competición. En este sentido, consideramos apropiado utilizar juegos modificados variados y motivantes en los que se desarrollen habilidades similares a la competición y en los que los jugadores puedan mejorar su capacidad física. A tal efecto, en un juego modificado para avanzar hacia la meta contraria, por ejemplo, sería conveniente aumentar el espacio y reducir el número de jugadores para mejorar la potencia aeróbica. O por ejemplo, reducir el tiempo de juego y premiar al equipo que más balones recupere en un juego de 3 contra 3 para mantener la posesión del balón, para mejorar así la táctica en la presión defensiva y la velocidad de reacción y la fuerza intermitente como demandas físicas (Cometti, 2002; Weineck, 2005).

3.5 El juego modificado, herramienta para el desarrollo de actitudes y medio de atención a la diversidad

Uno de los objetivos de la educación física y el deporte es la promoción de un estilo de vida saludable y el fomento de la práctica de actividad física. Además, el deporte también tiene una gran responsabilidad sobre la aceptación y superación de desigualdades de uno mismo y de los otros. Esto es todavía más importante en una sociedad tan heterogénea como la actual. Al respecto, los resultados de la investigación sugieren que la práctica de juegos modificados tiene un gran potencial en torno a la coeducación y el fomento de la práctica física a largo plazo (Van Acker, Carreiro da Costa, De Bourdeaudhuij, Cardon y Haerens, 2010).

Además, la correcta modificación deportiva unida a un buen planteamiento didáctico puede fomentar el desarrollo de competencias básicas sin descuidar los objetivos y los contenidos propios de cada deporte (Rodríguez, 2010). En este sentido, recomendamos al lector el trabajo de Velázquez (2001) por el valioso contenido de la obra en relación a la atención a la diversidad. Esta propuesta, presenta diferentes posibilidades de adaptación y modificación de las tareas de aprendizaje con el objetivo de atender íntegramente a las cuatro dimensiones de la diversidad: la motriz, la cognitiva, la psicoafectiva y la socioafectiva.

Los niños y niñas que comienzan a practicar un deporte poseen diferentes niveles de conocimientos y habilidades (Rovegno et al., 2001). No hay dos participantes iguales, por lo que desde aquí recomendamos adaptar los juegos modificados a los objetivos y contenidos de la enseñanza. Así, un profesional podría realizar modificaciones en el modelo didáctico empleado para adaptar el contenido a la competencia motriz de los aprendices, modificando las reglas de actuación de cada participante, por ejemplo. E incluso, podría modificar los juegos para mejorar la capacidad psicosocial y afectiva de los mismos. En este caso, obligando a realizar un número determinado de pases para favorecer la cooperación o limitando el número de puntos que puede alcanzar un jugador, para potenciar así la participación de los compañeros con menor nivel.

\section{Conclusiones}

El juego modificado en la enseñanza de deportes de invasión como el fútbol se presenta como una herramienta idónea y eficiente para el desarrollo del conocimiento y el rendimiento de juego del jugador. No obstante, una propuesta poco fundamentada o un planteamiento competitivo que no se adecue a las competencias del jugador, puede conllevar limitaciones en el proceso de aprendizaje.

De igual modo, se sugiere que el juego modificado se puede utilizar como contenido y evaluación de procedimientos técnico-tácticos en todas las edades y niveles. Siempre y cuando se realice una correcta modificación deportiva y se establezca un criterio fundamentado. Sin embargo, no se establecen unas directrices justificadas a seguir en la planificación y utilización del juego modificado. Y, se tiende a utilizar estrategias de modificación orientadas a la estructura del juego (zona de juego, número de jugadores, material, tiempo...) y no tanto a los principios y objetivos técnico-tácticos. Por tanto, solamente realizando estudios de juegos modificados concretos se podrá conocer su efecto sobre el aprendizaje, así como su funcionalidad sobre la competición.

\section{Bibliografía}

Alarcón, F., Cárdenas, D., Miranda, MT., Ureña, N. y Piñar, MI (2010). La metodología de enseñanza en los deportes de equipo. Revista de Investigación en Educación, 7, 91-103.

Bayer, C. (1992). La enseñanza de los juegos deportivos colectivos.

1 Barcelona: Hispano Europea.

Blomqvist, M., Vänttinen, T. y Luhtanen, P. (2005). Assessment of secondary school students' decision-making and game-play ability in soccer. Physical Education and Sport Pedagogy, 10(2), 107-119.

Bunker, D.J. y Thorpe, R.D. (1982). Amodel for the teaching of games in secondary schools. Bulletin of Physical Education 19(1), 5-9.

Cometi, G. (2002). La preparación física en el fútbol. Barcelona: Paidotribo.

Contreras, O.R., De la Torre, E. y Velázquez, R. (2001). Iniciación deportiva. Madrid: Síntesis.

Devís, J. y Peiró, C. (1992): Nuevas perspectivas en Educación Física: la salud y los juegos modificados. Barcelona: INDE.

Dodds, D., Griffin, L. y Placek, J. (2001). Chapter 2: Selected review of the literature on development of learner's domain -specific knowledge. Journal of teaching in physical education, 20,301-313.

Farrow, D., Pyne, D. y Gabbett, T. (2008). A Comparative Analysis of the Skill and Physiological Demands of Open and Closed Training Drills in Australian Football, International Journal of Sports Science and Coaching, 3, 485-495.

Fradua Uriondo, L. (1999): La visión de juego en el futbolista. Barcelona: Paidotribo.

French, K.E. y Thomas j.r. (1987). The relation of Knowledge development to children's basketball performance. Journal of Sport Psychology, 9, 15-32.

García López, L. M., Contreras, O., Penney, D. y Chandler, T. J. L. (2009). The role of transfer in games teaching: Implications in the development of the sports curriculum. European Physical Education Review, 15(1), 47-63.

Gabbett, T., Jenkins, D. y Abernethy, B. (2009).Game-Based Training for Improving Skill and Physical Fitness in Team Sport Athletes. 
International Journal of Sports Science \& Coaching, 4(2), 273283.

González, S., García, L. M., Contreras, O. R. y Sánchez-Mora, D. (2009). El concepto de iniciación deportiva en la actualidad. Retos: Nuevas tendencias en educación fisica, deporte y recreación, 15 , 12-20.

González Villora, S., García López, L. M., Contreras Jordán, O. R. y Gutiérrez Díaz del Campo, D. (2010). Estudio descriptivo sobre el desarrollo táctico y la toma de decisiones en jóvenes jugadores de fútbol (12 años). Revista Infancia y Aprendizaje, 33(4), 489-501.

González Vílora, S., García López, L. M., Pastor Vicedo, J. C. y Contreras Jordán, O. R. (2010). Estudio descriptivo sobre el desarrollo táctico y la toma de decisiones en jóvenes jugadores de fútbol (10 años). Revista de Psicología del Deporte, 20(1), 79-97.

Gréhaigne, J. F., Wallian, N. y Godbout, P. (2005). Tactical-decision learning model and students' practices. Physical Education and Sport Pedagogy, 10(3), 213-224.

Griffin, L.L., Dodds, P., Placek, J. y Tremino, F. (2001). Chapter 4 Middle School Students Conceptions of Soccer Their Solutions to Tactical Problems. Journal of teaching and physical education, 20, 324-340.

Griffin, L.L., Mitchell, S.A.y Oslin, J.L. (1997).Teaching Sport Concepts and Skills: A Tactical Games Approach. Champaign, II.: Human Kinetics.

Hartwig, T.B. y Naughton, G. (2007). A Movement-Analysis Comparison in Two Models of Junior Sport. Pediatric Exercise Science, 61-69.

Henninger, M.L., Pagnano, K.B., Patton, K., Griffin, L.L y Dodds, P. (2006). Novice volleyball players' knowledge of games, strategies, tactics, and decision-making in the context of game play. Journal of Teaching in Physical Education New Zealand, 39(1), 34-46.

Holt, J.E., Ward, P. y Wallhead, T.L. (2006). The transfer of learning from play practices to game play in young adult soccer players. Physical Education \& Sport Pedagogy, 11(2), 101-118.

Lago, C. (2002). La enseñanza del fútbol en la edad escolar. Sevilla: Wanceulen.

Lapresa, D., Amatria, M., Eguién, R., Arana, J. y Garzón, B. (2008). Análisis descriptivo y secuencial de la fase ofensiva en Fútbol 5 en la categoría prebenjamín. Cultura, Ciencia y Deporte, 8(3), 107116.

Lapresa, D., Arana, J., Garzón, E., Egüén, R. y Amatria, M. (2010). Adaptando la competición en la iniciación al fútbol: estudio comparativo de las modalidades de fútbol 3 y fútbol 5 en categoría prebenjamín. Apunts, 101, 43-56.

Lasierra, G. (1990). Aproximación a una propuesta de aprendizaje de los elementos tácticos individuales en los deportes de equipo.Apunts, 24, 59-68.

Lasierra, G. y Lavega, P. (1993): 1015 juegos y formas jugadas de

Launder, A. G. y Piltz, W. (2006). Beyond «Understanding» to skilful play in games, through Play practice. Journal of Physical Education New Zealand, 39(1), 47-57.

Light, R. (2006). Game Sense: Innovation or just good coaching?. Journal of Physical Education New Zealand, 39(1), 8-20.

Memmert, D. (2010). Testing of Tactical performance in youth elite soccer. Journal of sport science and medicine, 9, 199-205. iniciación a los deportes de equipo. Barcelona: Paidotribo.

Memmert, D. y Roth, K. (2007). The effects of non-specific and specific concepts on tactical creativity in team ball sports. Journal of Sports Sciences, 25(12), 1423-1432.

Mitchell, S. A., Oslin, J. L. y Griffin, L. L. (2003). Sport Fundations for Elementary Physical Education. A Tactical Games Approach.Human Kinetics. Champaign, IL.

Moreno, J. A. y Rodríguez, P. L. (1996). Aprendizaje Deportivo. Universidad de Murcia. Murcia.

Nevett, M., Rovegno, I. y Baviarz, M. (2001). Chapter 8. Fourth-grade children's knowledge of cutting, pass and tactics in invasion games after a 12-lesson units of instruction. Journal of teaching in physical education, 20, 389-401.

Placek, J. y Griffin, L. (2001). Chapter 9. The understanding and development of Learners's specific-knowledge: Concluding comments. Journal of teaching and physical education, 20, 402406.

Raab, M. (2007). Think SMART, not hard-a review of teaching decision making in sport from an ecological rationality perspective. Physical Education and Sport Pedagogy, 12(1), 1-22.

Rodríguez, D. (2001). El fútbol como herramienta para el trabajo de los valores y actitudes en la ESO según las Competencias Básicas. Retos: Nuevastendencias en educación física, deporte y recreación, 17, 67-71.

Rovegno, I., Nevett, M. y Babiarz, M. (2001). Chapter 5. Learning and teaching invasion-game tactics in 4th grade: Introduction and theoretical perspective. Journal of teaching in physical education, 20, 341-351.

Sans, A. y Frattarola, C. (1996): Entrenamiento en el fútbol base. Barcelona: Paidotribo.

Sans, A. y Frattarola, C. (1998): Fútbol base. Programa de entrenamiento para la etapa de tecnificación. Barcelona: Paidotribo

Sans, A. y Frattarola, C. (2009): Entrenamiento en el fútbol base. Programa de aplicación técnica $-1^{\circ}$ nivel (AT-1). Barcelona: Paidotribo.

Thorpe, R., Bunker, D. y Almond, L. (1986). A change in focus for the teaching of games. En Piéron, M. y Graham K. C. The 1984 Olimpic Scientific Congress Proccedings, 6. Sport Pedagogy. Champaign,IL. Human Kinetics.

Van Acker, R,. Carreiro da Costa, F., De Bourdeaudhuij, I., Cardon, G. y Haerens, L. (2010). Sex equity and physical activity levels in coeducational physical education: exploring the potential of modified game forms. Physical Education \& Sport Pedagogy, 15(2), 159173.

Velazquez, R. (2001). Iniciación a los deportes colectivos y atención a la diversidad. Actas del $2^{\circ}$ congreso internacional de Educación Física y Diversidad. Murcia: Universidad de Murcia.

Vera, G, Pino, J., Romero, C. y Moreno, M.I. (2007). Propuesta de valoración técnico-táctica mediante una situación de juego colectivo básico en el fútbol de iniciación. Retos. Nuevas tendencias en Educación Física, Deporte y Recreación, 12, 29-35.

Wein, H. (1995). Fútbol a la medida del niño. RealFederación Española de Fútbol. Madrid.

Weineck, J. (2005). Fútbol total. Entrenamiento físico del futbolista. Barcelona: Paidotribo.

Wilson, G (2002): A framework for teaching tactical games knowledge. Journal of Physical Education, Recreation and Dance. 73 (1), 20 26 y 56.

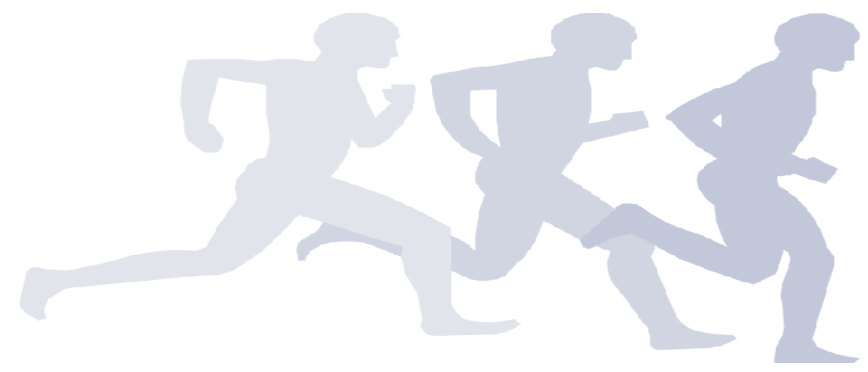

